\title{
Understanding long-term orbital debris population dynamics
}

\author{
Hugh G. Lewis \\ University of Southampton, Astronautics Research Group, Faculty of Engineering and Physical Sciences, \\ Boldrewood Innovation Campus 176/2041, Southampton SO16 7QF, UK, hglewis@soton.ac.uk
}

\begin{abstract}
Linear growth of the orbital debris population is observed in the results of many evolutionary models when they are used to simulate the effects of the widespread adoption of the Inter-Agency Space Debris Coordination Committee (IADC) debris mitigation guidelines. Such linear growth seemingly confirms the belief that adopting these debris mitigation guidelines will have substantial and positive benefits on the orbital debris population. However, this outcome is not expected from analyses of simple systems models. Either the systems model is too simplistic, or the linear growth of the debris population is actually the beginnings of exponential growth, which is difficult to discern over the typical analysis period. To resolve this ambiguity, the Debris Analysis and Monitoring Architecture to the Geosynchronous Environment (DAMAGE) model was used to perform ultra-long projections of the future debris population $\geq 10 \mathrm{~cm}$ in Low Earth Orbit (LEO) under highly optimistic debris mitigation conditions. The DAMAGE results showed that the linear growth rate observed for the first 200 years of the projection period was transient and the growth was exponential, even with the ongoing and widespread adoption of debris mitigation measures. The population growth was driven by the accumulation of orbital debris at altitudes between $1200 \mathrm{~km}$ and $1500 \mathrm{~km}$, even though simulated launch activity to that region was limited. Further, the results highlighted a tendency for high and sustained collision rates at altitudes below $700 \mathrm{~km}$, predominantly due to conjunctions between spacecraft and upper stages decaying through this region in observance with the "25-year rule". Overall, these results indicate that additional, and possibly new, measures to mitigate the effects of orbital debris may be needed, with particular emphasis on post-mission disposal and measures that protect the 1200-1500 km region in LEO.
\end{abstract}

\section{INTRODUCTION}

In 1978, Kessler and Cour-Palais warned of the hazards from orbital debris and the creation of a debris belt around the Earth unless measures to address the problem were implemented [1]. Despite this warning, over forty years of uninterrupted space activity since then has created a population of debris around the Earth that now threatens the safe operation of spacecraft and the long-term sustainable use of outer space [2]. There are an estimated 128 million particles between $1 \mathrm{~mm}$ and $1 \mathrm{~cm}, 900,000$ objects between $1 \mathrm{~cm}$, and $10 \mathrm{~cm}$, and 34,000 trackable objects larger than $10 \mathrm{~cm}$ in Earth orbit [3].

In response to the growing risks, The Inter-Agency Space Debris Coordination Committee (IADC) released mitigation guidelines that aim to limit the generation of orbital debris [4]. One guideline, often referred to as the "25-year rule", directs operators to adopt post-mission disposal (PMD) to limit the long-term presence of spacecraft and upper stages in the Low Earth Orbit (LEO) region. For reasons of fuel efficiency, spacecraft and orbital stages seeking to comply with this "25-year rule" tend to employ a maneuver that reduces the altitude of the orbit perigee without adjusting the altitude of the orbit apogee. This approach requires a single maneuver and results in an eccentric disposal orbit, with the perigee altitude selected to ensure re-entry into the Earth's atmosphere within 25 years. In 2013, an IADC study suggested that a $90 \%$ adoption rate of this measure could constrain the trackable population in LEO to linear growth under the assumption that launches continued at the prevailing rate [5].

Nevertheless, in some LEO altitude regimes other studies suggest that the number density of debris has already passed a tipping point where the rate at which new debris is added by collisions exceeds the rate at which debris decays due to atmospheric drag [6-7]. Studies by the US National Aeronautics and Space Administration (NASA) using a high-fidelity three-dimensional physical model indicate that growth of the trackable Earth satellite population in LEO is inevitable even if future launch activity is stopped [8].

The ambiguity in the interpretation of evolutionary model results is compounded by the commonly held misconception that these models enable accurate forecasts of the orbital debris population. It is not reasonable to use these models to make such forecasts. Instead, their primary purpose is to aid our efforts to anticipate significant 
events and trends in the population of orbital debris, to offer an understanding of the structural causes of the behavior of the Earth satellite population and to evaluate the responses to new space systems or debris mitigation measures. The typical complexity of fully 3D evolutionary models tends to preclude their use for robust analyses over periods longer than one or two centuries. This is an important limitation because many spacecraft and rocket bodies in LEO have orbital lifetimes much longer than this. Consequently, we lack a true understanding of the future behavior of the orbital debris population engendered by our space activities.

To gain insight into the true long-term behavior of the orbital debris population, under quite optimistic conditions with respect to existing debris mitigation measures, the Debris Analysis and Monitoring Architecture to the Geosynchronous Environment (DAMAGE) evolutionary model [9] was used to perform 1000-year projections of the future debris population $\geq 10 \mathrm{~cm}$ in LEO. The results were subjected to a preliminary analysis of the first 200 years of the projection period to illustrate the difficulties associated with the interpretation of the behavior of the orbital debris population over this relatively short time period. Analysis of the full projection period, based on expectations set by adopting systems thinking, was subsequently conducted in an effort to resolve the ambiguities.

\section{SIMULATION APPROACH}

DAMAGE is a high-fidelity three-dimensional physical model capable of simulating the evolution of future debris populations [9]. DAMAGE projections make use of a Monte Carlo approach to simulate future collisions. Within a given projection time step (here, 5 days), a random number is drawn and compared with the probability estimated for each pair of target and projectile objects found in close proximity (here, for a cube of side $10 \mathrm{~km}$ ). The Monte Carlo process requires multiple projection runs to be performed and analyzed before reliable and meaningful conclusions can be drawn from the outcome.

The simulation parameters used for this study correspond to the parameters used for the current reference case adopted by the IADC:

- A 1 February 2018 epoch with an initial population corresponding to all objects $\geq 10 \mathrm{~cm}$ residing within or crossing the LEO protected region;

- Launch traffic was assumed to be represented by the repetition of recent launches (taken from 1 January 2010 to 31 December 2017) with small random adjustments made to the exact launch date and orbital parameters to avoid artificially enhancing the likelihood of collisions on launch;

- New spacecraft and rocket upper stages were assumed to achieve a 90\% success rate with respect to postmission disposal, targeting an uncontrolled re-entry within 25 years by reducing the perigee altitude, as described above. No collision avoidance maneuvers were implemented.

- Vehicle passivation was assumed to be $100 \%$ successful such that no explosions were permitted within the projection period. Although explosions represent the dominant historical source of debris larger than 10 $\mathrm{cm}$, and continue to occur in reality, it was assumed for the study that all on-board sources of energy (e.g. propellant and batteries) were passivated. Consequently, only fragmentations due to collisions were permitted.

Here, 220 future projections of the $10 \mathrm{~cm}$ and larger debris populations from 1 February 2018 to 1 February 3018 were performed using DAMAGE, under the principle assumptions that the current pattern of launch activity and the implementation of post-mission disposal to orbits with lifetimes of 25 years would continue for the 1000-year period. The IADC disposal guideline permits operators to dispose of spacecraft and upper stages in so-called "graveyard" orbits at altitudes above the LEO region. From a purely cost-based perspective, this option is considered preferable for missions at or above about $1400 \mathrm{~km}$ because it has a lower propellant requirement compared with a maneuver to an orbit with a reduced lifetime. However, a parallel study by DAMAGE, which incorporated this option, resulted in a collision-cascading process in such a graveyard orbit. Consequently, a graveyard option was not permitted in this study. Simulations were conducted using 23 PC cores and were completed in 10 days.

\section{PRELIMINARY ANALYSIS}

The typical projection period for studies is 100 or 200 years. Most IADC studies since the 1990s have employed projection periods of 100 or 200 years because these were assumed to provide sufficient information about the causes of behavior of the debris population, without requiring excessive computer time. However, debris at altitudes 
above $700 \mathrm{~km}$ can have orbital lifetimes that are significantly longer than this. Consequently, our understanding of the true structural behavior of the debris population in LEO is poor, although this is not widely recognized.

\subsection{Expectations from the Current Situation}

Considering the fractional time spent within each $50 \mathrm{~km}$ altitude shell, objects at or below $1000 \mathrm{~km}$ altitude now account for $73.6 \%$ of the total spatial density in LEO. The peak spatial density in LEO is currently at an altitude of $800 \mathrm{~km}$ (see Fig. 1). In the period 2010-2017, worldwide launch activity accounted for an average 168.3 yearly spacecraft and upper stage deployments in LEO, with 90.4\% of all new objects injected into orbits at or below 1000 $\mathrm{km}$ altitude, and nearly three-quarters into orbits at or below $650 \mathrm{~km}$ (Fig. 1). Further, a substantial proportion of the existing orbital population and the future launch population are found in near-circular orbits (Fig. 2).

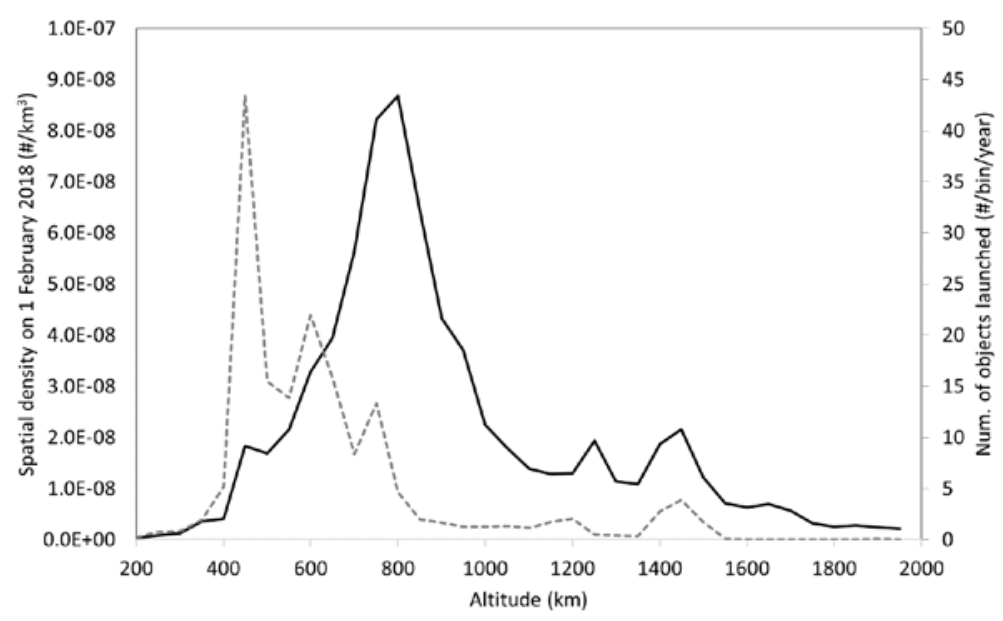

- Spatial density on 1 February 2018 ---- Number of objects launched per year (2010-2017)

Fig. 1. Spatial density of trackable objects in orbit on 1 February 2018, and average yearly count of objects launched to the space environment in the period 2010-2017. The spatial density and launch activity account for the fractional time, per orbital period, an object spends within each $50 \mathrm{~km}$ bin between $200 \mathrm{~km}$ and $2000 \mathrm{~km}$ altitudes.

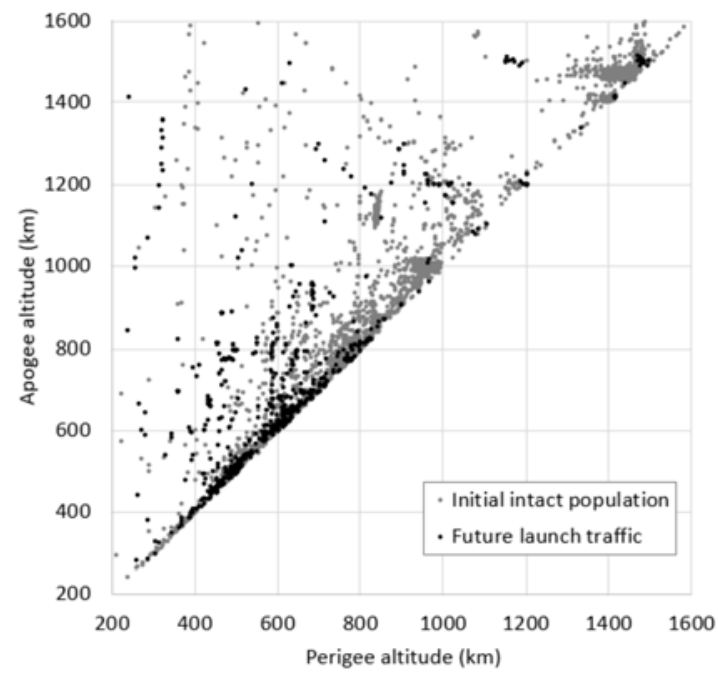

Fig. 2. Perigee and apogee altitudes of existing debris populations and recent space launches to LEO, not including objects residing in or launched to Geostationary Transfer Orbits (GTOs).

Based on our knowledge of the current situation and the target orbits for spacecraft and upper stages injected into the LEO region, a reasonable expectation is for the peak spatial density to remain at, or slightly below, an altitude of $800 \mathrm{~km}$. In addition, with a substantial proportion of new launches going into altitudes below $600 \mathrm{~km}$ altitude, we 
would likely consider the expectation that the growth of the population and the occurrence of catastrophic collisions in LEO could be limited due to natural compliance of these new objects with the "25-year rule". A catastrophic collision occurs when the ratio of impact kinetic energy to target mass exceeds 40,000 J/kg. The consequence of a catastrophic collision is the total fragmentation of the target (and the impactor). All of these expectations would be in-line with the conclusions drawn from the results of IADC and other studies [5].

\subsection{Analysis of a Typical 200-Year Projection Period}

If we restrict our view of the DAMAGE results to the first 200 years (Fig. 3), then the simulations indicate that the debris population generally grew at a constant, slow rate of 5.84 objects per year over this period in response to the launch activity and the widespread implementation of post-mission disposal. This lead to an overall increase in the orbital population of $13.88 \%$ by 1 February 2218 . Over the same period, the catastrophic collision rate remained constant at 0.23 catastrophic collisions per year. More than half (55.97\%) of all catastrophic collisions occurred at altitudes between $600 \mathrm{~km}$ and $900 \mathrm{~km}$, and nearly 90\% (89.51\%) occurred at or below $1000 \mathrm{~km}$ altitude.

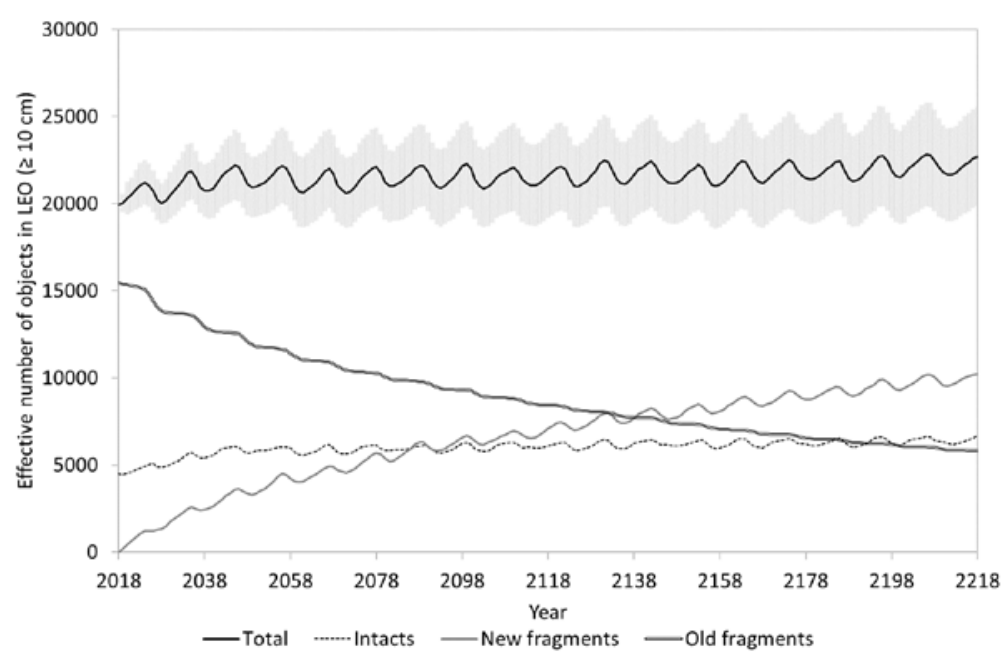

Fig. 3. Growth of future trackable object populations in LEO over a 200-year projection period. The filled area represents the \pm 1 -sigma error in the total effective number of objects.

A quantitative analysis therefore suggests that the application of the IADC post-mission disposal guideline and injection of launch traffic that is predominantly limited to altitudes below $1000 \mathrm{~km}$ would effectively stabilize the growth of the orbital debris population. A more pessimistic interpretation might still suggest that the population is constrained to a manageable, linear growth. In either case, there is no emergence of the much-popularized "Kessler Syndrome”. Even without the correspondence of the observed behavior with our expectations, or agreement with previous studies, this result would likely be interpreted as a success for debris mitigation measures. Given that our expectations have been met and that the results do corroborate our previous findings, it is not difficult to believe our understanding of the behavior of the orbital debris population is comprehensive and our foresight is reliable.

\subsection{Systems Thinking}

Systems thinking [10] can be applied to the orbital debris problem, enabling us to gain an understanding of the fundamental behaviours of the system. We first consider a simple physical system: a bath (Fig. 4a). In this system the water level is a stock (our quantity of interest) and the water inflow and water outflow are flows. These flows are controlled by a tap and a drain, respectively. We can draw this physical system using a simple stock and flow diagram (Fig. 4b) where the clouds represent other parts of the wider system that we are not including. In Fig. 4b the drain is also shown as a tap because it controls the outflow rate (it would not be a tap in the real world). 


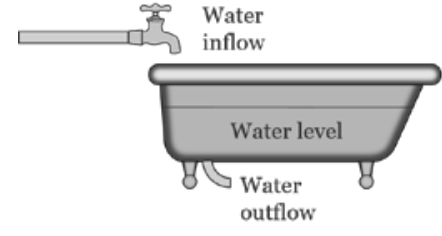

(a)

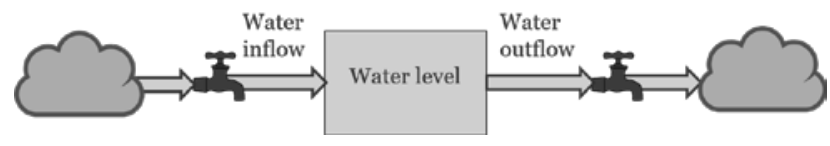

(b)

Fig. 4. Representation of a physical bath system (a) as a stock (water level) and flows that add to or take away from the stock (b).

If the drain is closed (i.e. there is no water outflow) and the tap is turned on, then the water level will rise (Fig. 5). If the drain is opened while the tap is still running, then the water level could continue to rise (water inflow is greater than water outflow), it could fall (water inflow is less than water outflow) or it could remain constant (water inflow is equal to water outflow). In the latter case, the water level is in dynamic equilibrium - water continues to enter the bath from the tap to replenish the water that is lost via the drain. If the tap is closed while the drain remains open, then the water level will fall. Systems thinking provides us with a way to understand the behaviour of the water level through time as it responds to changes in the flows. In this simple case, there is no feedback; the water inflow and the water outflow do not depend on the water level in the bath, so the water level will change linearly with time. In other words, the rate of change of the water level, $W$, is

$$
\frac{d W}{d t}=Q-D,
$$

where $Q$ is the water inflow rate and $D$ is the water outflow rate. Dynamic equilibrium occurs when $Q-D=0$. In other words, when $Q=D$.

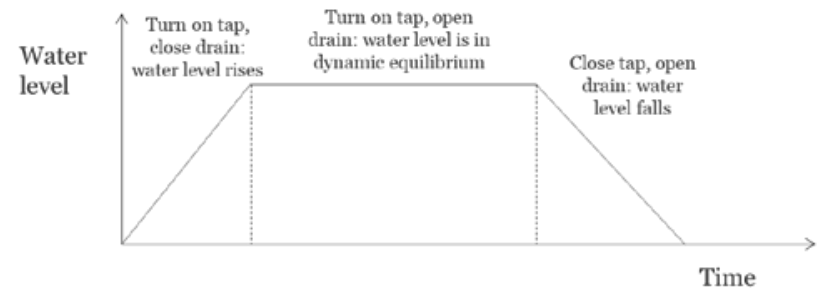

Fig. 5. Change in water level (stock) in the bath in response to different flows.

The orbital debris "system" has much in common with the bath example, but more closely resembles the human population system, where the number of people is the stock, births represent the inflow and deaths represent the outflow. The human population system has a reinforcing feedback (the birth rate depends on the number of people, modified by the fertility) and a balancing feedback (the death rate depends on the number of people, modified by the mortality). The reinforcing feedback leads to near-exponential growth whereas the balancing feedback leads to exponential decay. Therefore, there are only three fundamental types of behaviour for the human population system: exponential growth (birth rate $>$ death rate), exponential decay (death rate $>$ birth rate) and dynamic equilibrium (birth rate = death rate). In the case of the orbital debris system, the number of objects (e.g. that are trackable in LEO) is the stock (Fig. 6). There are two inflows: objects added through new launch activity and objects added as a consequence of collisions. The latter is a reinforcing feedback, which depends on the number of objects in the population. There is one outflow, atmospheric re-entry, which is a balancing feedback that also depends on the number of objects in the population. 


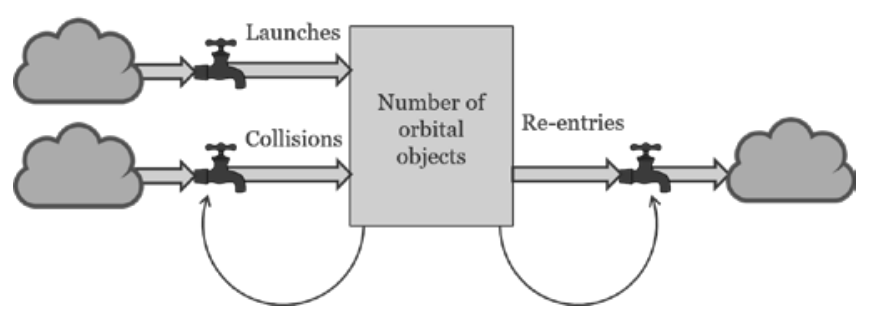

Fig. 6. Representation of the orbital debris system.

There are still only three fundamental types of behavior for this simple orbital debris system: exponential growth (launch rate + collision rate $>$ re-entry rate), exponential decay (launch rate + collision rate $<$ re-entry rate), and dynamic equilibrium (launch rate + collision rate $=$ re-entry rate). Linear growth is not a possible long-term behavior because the system moves into one of the other modes of behavior with just a small change to the number of objects.

This simple systems model has been implemented in the past as "Particles in a Box" (PIB) models with differential equations describing the flows [11],

$$
\frac{d N}{d t}=A-B N+C N^{2}
$$

where $N$ is the number of objects in the population, $A$ is the launch rate, $B N$ is the re-entry rate and $C N^{2}$ is the rate at which objects are added by collisions. The equilibrium populations are found easily from the roots of Eq. 2 . When $N=B / C$ the collision rate will be the same as the re-entry rate and Eq. 2 reduces to $d N / d t=A$, which suggests that linear growth is possible. However, this growth would inevitably lead to $N>B / C$, the sum of the non-linear terms in Eq. 2 would become non-zero and the fundamental non-linear behaviour would be restored. Although this model is an obvious approximation of the real orbital debris system, it can be readily extended to include multiple "boxes", and different types of debris. Nevertheless, the aggregated behaviour will follow the same principles as a single "box".

Systems thinking therefore suggests that the linear growth observed in the results of the 200-year projection (Fig. 3) is actually the beginnings of an exponential growth in the population of orbital debris. The constant collision rate over the 200-year projection is also deceptive and is likely to be similarly exponential. Consequently, the conclusions that would be typically drawn from such a study (as suggested above) are also likely to be incorrect.

\section{FULL RESULTS AND ANALYSIS}

Considering the full 1000-year projection period (Fig. 7), the same DAMAGE simulations show that the debris population actually grows at an increasing rate over the projection period, in-line with the expectations from systems thinking. In the last 200-years of the full projection period, the debris population grew at a rate of 48.28 objects per year, more than eight times faster than during the first 200-year period. By 1 February 3018, the orbital population had increased by $134.10 \%$ from its initial level.

Analysis of the spatial density in the altitude regime below $1000 \mathrm{~km}$ shows very little variation over the projection period (average change over all altitudes $\leq 1000 \mathrm{~km}=17.03 \%$ ) indicating that new launches and the creation of new collision fragments is balanced in this region by debris decaying due to atmospheric drag (Fig. 8). In contrast, the spatial density increases at altitudes above $1000 \mathrm{~km}$ by $368.16 \%$ (average over all altitudes $>1000 \mathrm{~km}$ ) through the projection period. At $1350 \mathrm{~km}$, in particular, the spatial density at the end of the projection period is 8.3 times higher than the spatial density there on 1 February 2018, despite this being one of the least congested altitudes in LEO at the outset and with relatively few launches into this region (Fig. 1). The increasing debris population above $1000 \mathrm{~km}$ is the cause of the overall accelerating population growth seen in Fig. 7. 


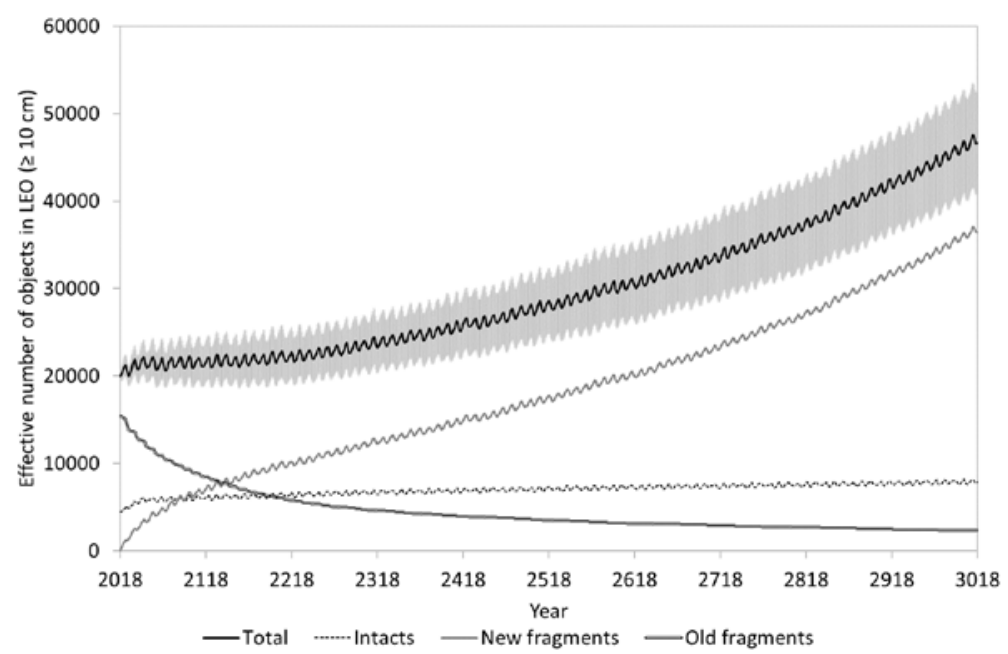

Fig. 7. Growth of future trackable object populations in LEO over a 1000-year projection period. The filled area represents the \pm 1 -sigma error in the total effective number of objects.

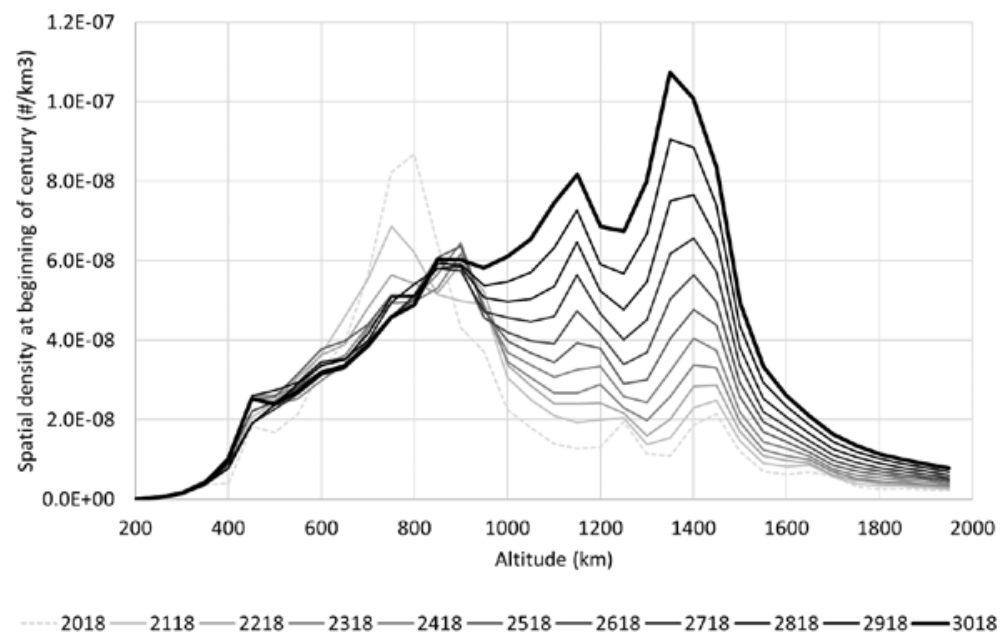

Fig. 8. Debris spatial density at the beginning of each century of the projection period for objects $10 \mathrm{~cm}$ and larger.

Catastrophic collisions at or below an altitude of $1000 \mathrm{~km}$ tended to occur at a nearly constant rate of one event every 3.9 years throughout the projection period (computed from observations made at the beginning of each century of the projection, Fig. 9). Conversely, the expected interval between catastrophic collisions occurring at altitudes above $1000 \mathrm{~km}$ decreased from 49.33 years to 6.62 years by the end of the projection period, despite less than $10 \%$ of new launches targeting this region and in spite of the relatively low spatial density there on 1 February 2018. The time taken to halve the interval between catastrophic collisions in this region is 200-300 years (i.e. longer than the typical projection period used in simulation studies). Hence, it becomes possible to envisage a time - by extrapolating the trend in Fig. 9 thousands of years in the future - when the expected interval between catastrophic collisions is measured in minutes, rather than years, and where the environment would experience a manifestation of the "Kessler Syndrome" not unlike the depiction in the movie "Gravity".

After experiencing more than a seven-fold (7.38) increase in the catastrophic collision rate at an altitude of $1400 \mathrm{~km}$, this specific region became the principal location for collisions in LEO by the end of the projection period. In spite of this, nearly three-quarters (72.23\%) of the total number of catastrophic collisions occurred at or below an altitude of $1000 \mathrm{~km}$, and nearly one-third (31.26\%) at or below $650 \mathrm{~km}$ (see Fig. 10). 


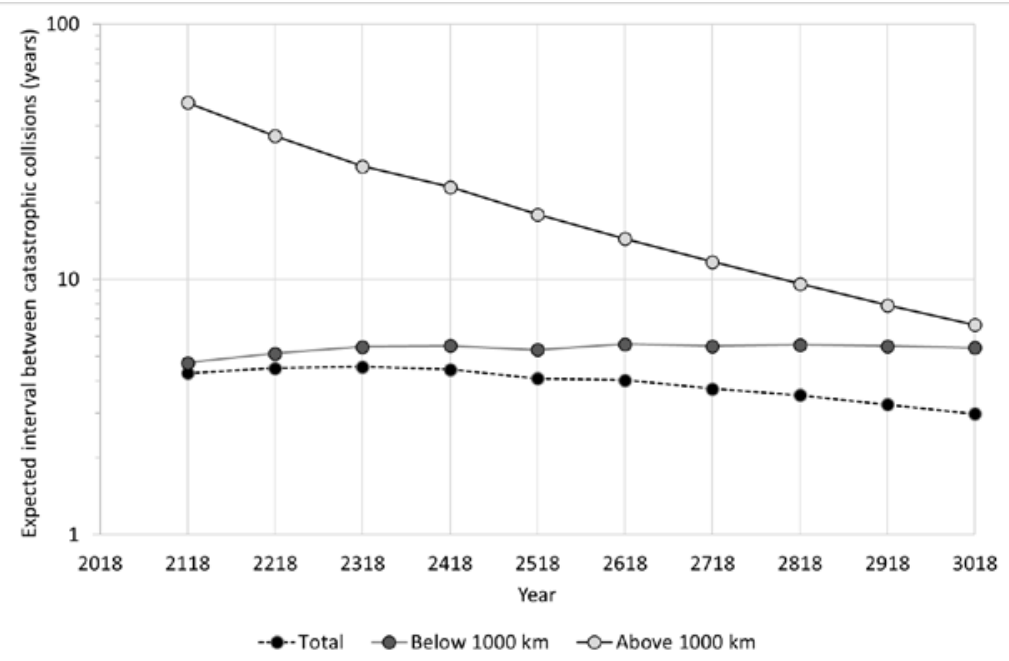

Fig. 9. Expected interval between catastrophic collisions in LEO calculated at the beginning of each century of the projection period.

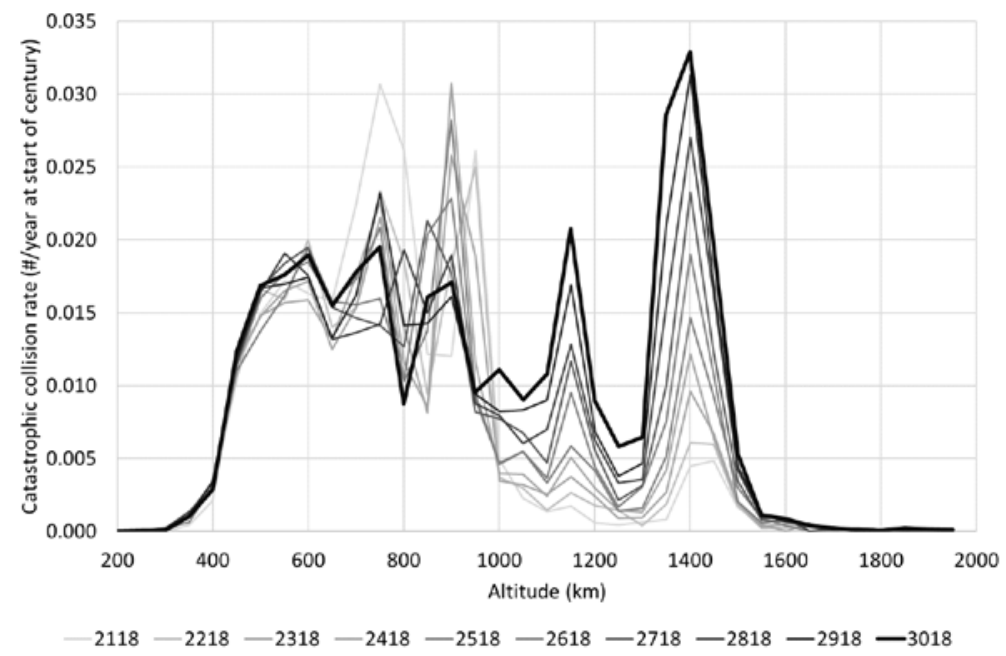

Fig. 10. Catastrophic collisions rate calculated at the beginning of each century of the projection period.

It could be argued that a higher compliance with the 25-year rule by spacecraft and upper stages (i.e. > 90\%) might limit the population growth at altitudes near $1400 \mathrm{~km}$ but a detailed analysis of the results indicates that the number density there at the beginning of the projection period is already at the critical level. The population remains in dynamic equilibrium without the addition of new launch traffic. Consequently, even a few non-compliant spacecraft or upper stages deployed to this regime, or a missed collision avoidance maneuver there, might initiate the runaway growth observed in the study even with near-perfect post-mission disposal behavior. Furthermore, the use of graveyard orbits above LEO is also likely to be unsustainable. All DAMAGE simulations of a 1000-year projection period that included this graveyard option resulted in runaway population growth, driven by an accelerating collision rate between objects in the graveyard orbits.

Notably, the orbits of spacecraft and upper stages originally deployed to higher altitudes will intersect the region below $700 \mathrm{~km}$ altitude following maneuvers to comply with the "25-year rule". Given the assumption of a $90 \%$ postmission disposal success rate, it is evident that $90 \%$ of all spacecraft and upper stags launched to high altitudes in LEO would subsequently spend 25 years crossing altitudes below $700 \mathrm{~km}$; a region that was also the target of most new launches. A detailed analysis of the results revealed that two-thirds (66.24\%) of all catastrophic collisions involving an intact primary and an intact secondary, also comprised at least one spacecraft or upper stage that had maneuvered to be fully compliant with the "25-year rule" (1181 collisions from 2613 catastrophic collisions observed in 10 Monte Carlo runs involving exclusively intact objects). Nearly one-quarter (24.28\%) of all 
catastrophic collisions involving exclusively intact objects were the result of conjunctions where both the primary and secondary objects had maneuvered to orbits with lifetimes less than 25 years.

The orbits of the intact primary and secondary objects experiencing catastrophic collisions are shown in Fig. 11. The cluster of points between $400 \mathrm{~km}$ and $700 \mathrm{~km}$, and above the diagonal are not seen in the launch traffic or the initial population (Fig. 1) and represent objects that have maneuvered to eccentric disposal orbits, with apogee altitudes higher than the perigee altitudes.

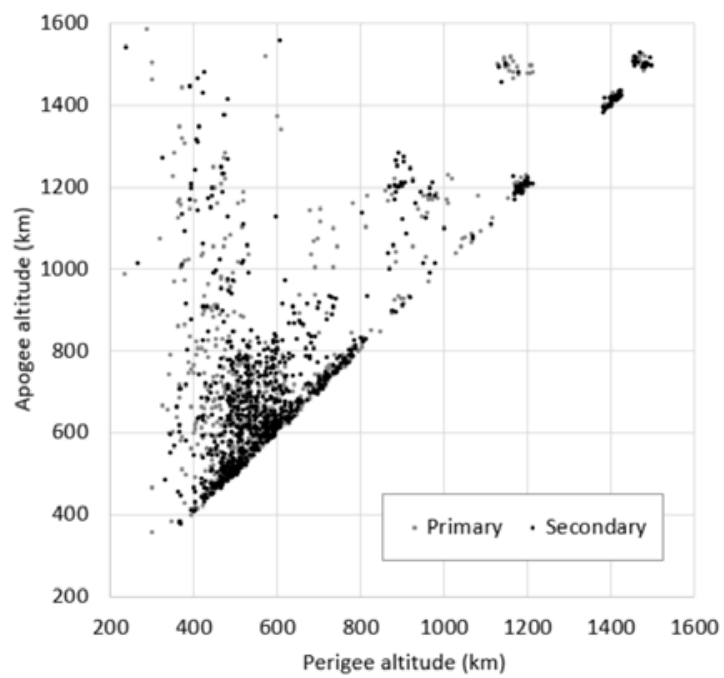

Fig. 11. Orbits of intact objects involved in catastrophic collisions in LEO. Results from 10 Monte Carlo runs.

The impact of post-mission disposal maneuvers on collision activity below $700 \mathrm{~km}$ was appreciated even before the introduction of the IADC post-mission disposal guideline for objects in LEO. Referring to the implementation of deorbit rules in a 2001 simulation study [12] said, "The act of reducing perigee of all intacts at end-of-life increases the time spent at the lower altitudes and also increases the likelihood of collision at those low altitudes."

Evidently, the level of concern was insufficient to prevent the adoption of the "25-year rule" or the continued drive to increase the level of compliance with it [13]. Nevertheless, this self-induced collision activity will have been a characteristic of every simulation study featuring an implementation of the de-orbit rule. Thus, an important reminder emerges from this 1000-year simulation: in absolute terms, compliance with the "25-year rule" in the higher LEO regime leads to a substantial number of catastrophic collisions that dominate the collision activity in the lower LEO altitude regime, and maintain the orbital population in a dynamic equilibrium. In other words, behavior that is widely accepted to be responsible actually drives and sustains the collisions it was intended to prevent. Furthermore, increasing the post-mission disposal compliance rate to values higher than the simulated $90 \%$ is unlikely to prevent runaway population growth in the higher altitude regime but might initiate exponential population growth below $1000 \mathrm{~km}$ by increasing the population there above the critical level. In the conclusions to their study, [12] noted that "Enhanced collisional activity at the lower altitudes... decreases as the PMD time decreases, since less time at lower altitudes reduces collision probability." Consequently, serious consideration should be given to an investigation of alternative de-orbit "rules".

\section{CONCLUSIONS}

The results show that despite the assumed low number of launches to altitudes above $1000 \mathrm{~km}$, widespread adoption of the 25-year rule and a relatively low collision rate at the outset, the debris population there grows at an accelerating rate. The increasing population ultimately leads to a substantially higher spatial density and a correspondingly higher collision rate there, compared with any other region in LEO. In the simulations, altitudes between $1200 \mathrm{~km}$ and $1500 \mathrm{~km}$ experienced the most rapid population growth and decrease in the expected interval between catastrophic collisions.

The results also indicated that the assumed post-mission disposal behavior contributed substantially to the collision activity, and sustained the debris population in dynamic equilibrium, in orbital altitudes below $700 \mathrm{~km}$. This system 
response will be evident in all simulation studies containing an implementation of a post-mission de-orbit rule, but its importance has possibly been overlooked. Ultimately, it means that something that is widely acknowledged to be a valuable orbital debris mitigation measure - a measure incorporated into debris mitigation plans that are accepted by many regulators - is based on a biased understanding of the dynamics of the orbital debris population.

A possible solution may be to maneuver spacecraft and upper stages to disposal orbits at lower altitudes than assumed, with residual lifetimes substantially shorter than 25 years. Lower altitudes have higher values of the critical spatial density, so can sustain a larger population of objects [6]. However, this needs to be achieved in conjunction with a higher compliance rate at altitudes above $1000 \mathrm{~km}$, or with a method to actively remove spacecraft and upper stages from this LEO regime. These solutions have important financial implications for spacecraft operators and introduce new and substantial challenges for regulators. In addition, advances in active removal technologies and in-orbit demonstrations are needed. Whatever the proposed solution, new simulation studies would be needed to provide a robust and reliable assessment before any implementation.

\section{ACKNOWLEDGEMENTS}

The author would like to thank colleagues from the Space Debris Office of the European Space Agency for the provision of and permission to use the MASTER population and launch traffic for this work.

\section{REFERENCES}

1. Kessler, D.J. and Cour-Palais, B. Collision frequency of artificial satellites: The creation of a debris belt, J. Geophys. Res. Vol. 83 (A6), pp. 2637-2646, 1978.

2. The White House. "Policy Directive 3 - National Space Traffic Management Policy,” Washington DC, 2018.

3. European Space Agency. "Space debris by the numbers," Available at https://m.esa.int/Our_Activities/Space_Safety/Space_Debris/Space_debris_by the_numbers, accessed 1 November 2019.

4. Inter-Agency Space Debris Coordination Committee. "IADC Space Debris Mitigation Guidelines,” IADC-0201, 2007.

5. Liou, J.-C. et al. "Stability of the Future LEO Environment - An IADC Comparison Study," $6^{\text {th }}$ European Conference on Space Debris (ESA SP-723), 2013.

6. Kessler, D.J. and Anz-Meador, P.D. "Critical number of spacecraft in Low Earth Orbit: Using satellite fragmentation data to evaluate the stability of the orbital debris environment," $3^{\text {rd }}$ European Conference on Space Debris (ESA SP-473), pp. 265-272, 2001.

7. U.S. National Research Council. "Limiting Future Collision Risk to Spacecraft: An Assessment of NASA's Meteoroid and Orbital Debris Programs,” Washington DC, 2011.

8. Liou, J.-C. and Johnson, N.L. Risks in space from orbital debris, Science, Vol. 311 (5759), pp. 340-341, 2006.

9. Lewis, H.G. et al. Effect of thermospheric contraction on remediation of the near-Earth space debris environment, J. Geophys. Res. Vol. 116 (A2), A00H08, 2011.

10. Meadows, D. Thinking in Systems, Vermont, US: Chelsea Green Publishing Company, 2008.

11. Talent, D.L. Analytic Model for Orbital Debris Environmental Management, J. Spacecraft and Rockets, Vol. 29 (4), pp. 508-513, 1992.

12. Krisko, P.H. et al. Evolve 4.0 orbital debris mitigation studies, Adv. Space Res., Vol. 28 (9), pp. 1385-1390, 2001.

13. European Space Agency Space Debris Office. “ESA’s Annual Space Environment Report,” Issue 3.2, 17 July 2019. 